\title{
Mode-switching: A new technique for electronically varying the agglomeration position in an acoustic particle manipulator.
}

\author{
Peter Glynne-Jones $^{1 *}$, Rosemary J. Townsend ${ }^{1}$, Nicholas R. Harris ${ }^{2}$, Andy W.J. Cranny ${ }^{2}$, \\ Martyn Hill ${ }^{1}$ \\ 1) School of Engineering Sciences \\ ${ }^{2)}$ Electronics and Computer Science \\ University of Southampton, UK \\ * Corresponding author: School of Engineering Science, University of Southampton, \\ University Road, Southampton, SO17 1BJ. Tel. +44 23805922339. \\ email: P.Glynne-Jones@soton.ac.uk
}

\begin{abstract}
Acoustic radiation forces offer a means of manipulating particles within a fluid. Much interest in recent years has focussed on the use of radiation forces in microfluidic (or "lab on a chip") devices. Such devices are well matched to the use of ultrasonic standing waves in which the resonant dimensions of the chamber are smaller than the ultrasonic wavelength in use. However, such devices have typically been limited to moving particles to one or two predetermined planes, whose positions are determined by acoustic pressure nodes/antinodes set up in the ultrasonic standing wave. In most cases devices have been designed to move particles to either the centre or (more recently) the side of a flow channel using ultrasonic frequencies that produce a half or quarter wavelength over the channel respectively.

It is demonstrated here that by rapidly switching back and forth between half and quarter wavelength frequencies - mode-switching - a new agglomeration position is established that permits beads to be brought to any arbitrary point between the half and quarter wave nodes. This new agglomeration position is effectively a position of stable equilibrium. This has many potential applications, particularly in cell sorting and manipulation. It should also enable precise control of agglomeration position to be maintained regardless of manufacturing tolerances, temperature variations, fluid medium characteristics and particle concentration.
\end{abstract}

PACS codes: 43.25.Qp, 43.20.Ks

Keywords: ultrasonic particle manipulation, acoustic radiation force, layered resonators, microfluidics, mode-switching

\section{Introduction}

An acoustic discontinuity within a sound field will generally experience a force due to the scattering of the wave by the discontinuity. Such forces occur in both progressive waves and standing waves and are due to a nonlinear interaction [1]. The acoustic radiation forces generated on small particles (relative to the wavelength) within an ultrasonic standing wave are significantly higher than forces produced within progressive waves, and systems based around ultrasonic standing waves are of interest for particle manipulation in 
microfluidic (or "lab on a chip") systems. Such systems are particularly promising for the handling of micron-scale biological cells for applications such as preparation [2,3] or fractionation $[4,5]$ of samples prior to analysis, the formation of aggregates for cell interaction studies [6], or for biosensor enhancement [7, 8]. Although high-intensity ultrasound can be used to destroy cells [9], there is now a significant body of evidence to show that, at the energy levels required for particle manipulation, cell viability is maintained $[10,11]$ along with the propensity of the cells to proliferate following exposure [12].

A variety of approaches to manipulation have been reported, including the use of focussed ultrasound [13] or near-field effects [14] to trap particles, the use of two or more transducers to modulate the standing wave field [15], or the use of plate waves coupled into the containing fluid to excite the field [16]. However, the use of a simple planar layered resonator with a single transducer $[1,17,18]$ offers the simplest approach to establishing a predictable USW suitable for particle movement.

Planar USW systems may employ resonators that are larger than a wavelength and contain multiple pressure nodal planes [19], but for microfluidic scale devices, a resonant cavity with a thickness in the direction of sound propagation that is lower than the operating wavelength is typically employed $[2,20]$. Such sub-wavelength resonators generally rely for their operation on precise positioning of the pressure node, to which particles will migrate. In these systems the ability to design a resonator that will operate with a high efficiency and have the required acoustic mode shapes is critical. In order to keep temperature increases within acceptable limits, these devices must be operated at modes which are associated with high acoustic energy densities in the fluid. Further, the size of microfluidic resonators typically places tight tolerances on device manufacture and the expense of many microfabrication methods precludes an iterative experimental approach to their development. Hence, the ability to design sub-wavelength resonators that are efficient, robust and have the appropriate acoustic energy distribution is important.

Modelling of planar multilayered resonant structures allows appropriate layer thicknesses to be calculated for placement of pressure nodes at known positions within the fluid layer [18, 21], including placing the pressure minimum at the reflector boundary [22]. As an example, Figure 1 shows a multilayer structure, typically used as the basis for an ultrasonic manipulator. It is required to position a pressure node at a predetermined point in the fluid layer. Previously this has been achieved by adjusting the thicknesses of the various layers to achieve an acceptable pressure profile within the fluid layer. Particles will generally (see Gröschl [1] for conditions and counter-examples) be transported towards pressure nodes within the field. A simple case of this is where the fluid layer is made to be half a wavelength deep at the operating wavelength (Half wave device). This would give a pressure anti-node (velocity minimum) at the reflector and carrier layer boundaries, leading to a pressure node in the centre of the fluid layer. An alternative strategy would be to generate a resonance in the reflector layer which can have approximate pressure release boundary conditions, and under these conditions the node in the fluid layer would be at the fluid/reflector boundary (Quarter wave device). Designs usually aim to optimize the response for a given node (although it is possible to design for multiple conditions [23] whereby particles can be moved from the centre to a surface, for example) but for accurate positioning of that node, system constraints are quite severe, requiring close tolerances of the thicknesses of the various layers, and good knowledge of their material parameters. Although this can be mitigated to a certain extent by choosing the least sensitive solutions to the controlling equations [24] a solution that allowed adjustment of the position of the node without requiring the remaking of the device would be a significant development in these types of system. This paper reports a technique that allows exactly this adjustment to 
be achieved, by electronic variation. The chamber is driven alternately at its half wave and quarter wave frequencies, and by varying the duty cycle and amplitude, it is possible to move particles to an arbitrary position between these two limits. Switching between modes has been reported by Petersson [25 p.51], however its use there was as a means of effecting fractionation by exploiting the different velocities of particles of different sizes as they moved between node positions. Modulation of drive frequencies is described by Manneburg et al. [26], where both high $(1 \mathrm{kHz})$ frequency modulation is used to average the force profile from several similar acoustic modes, and also low $(0.5 \mathrm{~Hz})$ frequency modulation to achieve particle transport. Mode switching is presented here in the context of a conventional planar resonator, such as that shown in Figure 1, but should be equally applicable to switching between appropriate modes in a laterally excited resonant chamber such as those described by Petersson et al. [5, 20, 25].

\section{Modelling force profiles due to mode-switching}

As described above, the aim of this work is to generate a time-averaged force profile by switching between the force profiles produced by two modes with different nodal positions. The switching frequency used will determine the frequency and amplitude of particle oscillation about the effective agglomeration position and the frequency must be sufficiently low for transient effects in the drive circuitry, transducer and resonant field itself to be short in comparison with the switching period. In addition, for the timeaveraging to be a matter of simple, weighted superposition, the change in force as a particle moves within each switching period must be small in comparison with the force itself. This condition is considered in more detail in the following.

For a planar resonator of the type illustrated in Figure 1, the force profile through the fluid layer for both the quarter and half-wave modes may be expressed as [1]:

$$
F=A \sin (2 k(z+a))
$$

where $A$ is the force amplitude, $a$ the node position offset (see below), $z$ the distance from the carrier-layer/fluid boundary, and $k$ the wave number of the ultrasonic standing wave given by

$$
k=\frac{2 \pi f}{c}
$$

where $c$ is the speed of sound in the liquid, and $f$ the frequency of the ultrasound.

Figure 2 shows the force profiles through the fluid layer for an idealised device in which the wavelengths of the quarter and half-wave modes are exactly one quarter and one half of the fluid layer height. The fluid layer is $200 \mu \mathrm{m}$ thick, force amplitudes both $1 \mathrm{pN}$, and $a=0$. The bottom of the graph corresponds to a point where the carrier layer meets the fluid, and the top the boundary with the reflector. A positive force is a force towards the reflector. The circles show the position of the pressure node in the standing wave - in the absence of other forces a particle will be moved towards these points. With the quarterwave mode a particle will experience a force at any point in the fluid layer towards the reflector, while with the half-wave mode particles will experience a force towards the centre of the fluid layer.

In a real device, the chamber does not have to be exactly one half or quarter wavelength (for instance to force a particle against the reflector it is useful to have the 
quarter-wave node located some distance into the reflector); thus the pressure nodes can be offset from the ideal position shown here, modelled by the inclusion of $a$ in equation 1 above. Also, under the action of gravity, the effective equilibrium position will be offset from the pressure node.

We now consider switching the drive to the transducer rapidly between the frequencies that produce the half and quarter-wave modes - effectively frequency modulating the drive signal with a square wave. By varying the mark to space ratio of the modulating square wave the proportion of quarter wave to half wave can be varied. If the modulating frequency is high enough that the particle does not have time to move to an area of significantly different force before switching to the other mode then the macroscopic effect can be approximated as being due to a single time-averaged force profile given by

$$
F_{Q H}=\alpha F_{Q}+(1-\alpha) F_{H}
$$

where $\alpha$ is the proportion of time the quarter wave is on, and $F_{Q}$ and $F_{H}$ are the acoustic force profiles produced by the quarter- and half-wave modes respectively.

This modulating frequency condition will be met (and should be met for both the quarter and half-wave modes) if

$$
\frac{d F}{d z} \Delta z<<F
$$

that is, if the change in force experienced by a particle as it moves under the acoustic force though the time period of the modulation is much less than the force it initially experiences.

In a microfluidic system with low Reynold's number the motion of the particle will be dominated by viscous drag (this approximation can be seen to be valid in the experiments described below, where the maximum time period is found to be of the order of a tenth of a second, while the terminal velocity, will be approached in a few tens of microseconds), leading to a terminal velocity, given by Stokes's formula, of

$$
v_{t}=\frac{F}{6 \pi \mu r}
$$

where $\mu$ is the dynamic viscosity and $r$ the bead radius. Thus the distance moved by the bead during the modulation time period is approximated by

$$
\Delta z=v_{t} t
$$

where $t$ is the time period of the modulation.

Substituting equations 1 and 6 into equation 4, we have

$$
2 k A \cos (2 k(z+a)) \frac{F}{6 \pi \mu r} t<<F
$$

Taking the maximum value of the $\cos ()$ term as 1 (which corresponds to a particle at a point in the fluid where the force is at a maximum), and substituting equation 2 , we have

$$
t<\frac{3 \mu r c}{2 f A}
$$

The approximations made in this derivation allow for worst case situations (the whole of the time period is devoted to one mode, and the bead is at a position of maximum force), and in practice we envisage that the technique may still be effective if time period longer than that indicated by equation (8). The equation is still useful, however, as it provides a simple means of ensuring that equation (3) will be valid throughout a given device. 
Figure 3 plots the simulated force profiles for a range of different values of $\alpha$ in the absence of gravitational/buoyancy force. It can be seen that new effective equilibrium positions (agglomeration positions) are formed, lying at any point between the quarter- and half-wave nodes.

Figure 4 plots the agglomeration position versus $\alpha$. The relative strengths of the two modes (which will also depend on the acoustic losses found at each frequency) can be varied by modifying the drive voltage during the respective parts of the cycle: Lines (b) and (c) illustrate the effect of having a half-wave mode stronger than the quarter. Gravity is not included in (a), (b) and (c) - which could represent a device turned on its side; the simulation of line (d) includes a $0.1 \mathrm{pN}$ force from gravity acting towards the carrier layer. In the absence of gravity, the agglomeration position depends only on the ratio $F_{H} / F_{Q}$ and $\alpha$.

Comparing cases (b) and (d) which both have $\mathrm{F}_{\mathrm{H}}=2 \mathrm{~F}_{\mathrm{Q}}$ it can be seen that the effect of gravity is most pronounced when there is just enough of the half-wave to move the agglomeration position away from the reflector (around 80\% quarter-wave in this case). This is due to the acoustic forces working against each other, and producing a weaker resultant $\mathrm{F}_{\mathrm{QH}}$.

It should be noted that the new agglomeration position is effectively a position of stable equilibrium - if the particle is displaced from the agglomeration position, it experiences a restoring force towards that position. However, feedback and control may be useful to fine-tune the agglomeration position to counteract positional uncertainties produced by finite manufacturing tolerances of a device.

\section{A microfluidic chamber demonstrating mode switching}

To demonstrate the effect of mode-switching experimentally, the chamber shown in Figure 5 was constructed; Table 1 lists its key dimensions in the column headed "circular chamber”. It comprises a PZT transducer (Ferroperm, PZT26) glued (Epotek 301 epoxy) to a carrier layer milled from Macor (a machinable ceramic material). A steel spacer fixes the height of the channel, which is bounded by a moulded silicone elastomer gasket. The reflector layer is formed from a microscope slide of soda-glass. A PMMA manifold permits fluidic connectors to deliver a bead / water mixture to the chamber. The beads are $10 \mu \mathrm{m}$ diameter fluorescent polystyrene beads (Polysciences Inc., Fluoresbrite microspheres). The acoustic properties of the chamber were designed using the process described by Hill et al.[24]. The modelling predicted the frequencies of the half- and quarter-wave modes, and their associated node positions as illustrated in Figure 6.

To measure the strengths of each of the two modes, a polystyrene bead was levitated and the threshold voltage where the bead begins to sediment recorded, in a manner similar to the method described by Martin et al. [8]. At the point where the bead is just about to begin to sediment the acoustic force is exactly balanced by the gravitational and buoyancy forces. For the $10 \mu \mathrm{m}$ diameter beads of density $1.055 \mathrm{~kg} / \mathrm{m}^{3}$ [8] the effective gravitational force (i.e. subtracting the effects of buoyancy from the gravitational body force) is $0.283 \mathrm{pN}$. The beads were found to drop at amplitudes of $9.5 \mathrm{Vpp}$ and $5.6 \mathrm{Vpp}$ for the quarter and half-wave modes respectively. Since the acoustic force is proportional to the square of the drive voltage amplitude, this means that operating the device at $17 \mathrm{Vpp}$ the force amplitudes take the values: $\mathrm{F}_{\mathrm{Q}}=0.906 \mathrm{pN}$ and $\mathrm{F}_{\mathrm{H}}=2.61 \mathrm{pN} \pm 8 \%$. The estimated error associated with these values stems from the difficulty in distinguishing between a particle sedimenting very slowly and one still levitating. Further complicating measurements is the fact that there are lateral variations in the strengths of the acoustic field due to lateral acoustic modes [27-29]. These variations can cause particles to migrate slowly during an experiment into areas of different field strength. To maintain consistent results, all the data 
presented here is for beads in an area, around $100 \mu \mathrm{m}$ in diameter, in the centre of the fluidic chamber. The magnitude of the acoustic forces predicted by the one-dimensional model was found to be larger than that found experimentally (see below), perhaps due to poor estimates of damping or due to energy being transmitted into structural and lateral modes.

To measure the height at which beads were held, a marked scale on the focusing adjustment of a Motics (B3 professional series, 10x objective) microscope was used. After compensating for the refractive effect of the fluidic and reflector layers, an accuracy of about $\pm 4 \mu \mathrm{m}$ was attainable. Beads were illuminated with a $405 \mathrm{~nm}$ violet laser to permit easy visualisation. To drive the transducer a function generator (TTi TG1304) was used. Using a viscosity of $10^{-3}$ Pa.s and sonic velocity of $1480 \mathrm{~ms}^{-2}$ for water, it is found that for the average force equation (3) to hold, the modulation frequency must be much greater than $1.0 \mathrm{~Hz}$. A modulation frequency of $100 \mathrm{~Hz}$ was chosen to meet this condition.

The position of the quarter wave node was estimated to be about $2 \mu \mathrm{m}$ into the reflector - found by adjusting model parameters to match the observed particle drop height in the levitation experiment (since the bead touches the reflector when its centre is $5 \mu \mathrm{m}$ from the reflector it is not possible to observe the node height directly). The position of the half-wave node was found to be $94 \mu \mathrm{m}$ from the carrier-layer/fluid boundary - this was derived from the observed bead position, and allowing for the effect of gravity. Figure 7 shows the predicted force profiles using the experimentally determined force amplitudes and node positions. It is interesting to note that in the half-wave mode there is a pressure anti-node near the bottom of the device: particles near the bottom of the fluid layer will be forced downwards towards the carrier layer, and not reach the nodal position near the centre of the fluid layer - this does not interfere with the demonstration of the mode-switching, as the quarter-wave mode can be used to raise particles above the anti-node prior to beginning experiments.

Having estimated the force profiles due to each mode, equation (3) can be used to predict the agglomeration position for different values of $\alpha$. Figure 8 shows the experimental results for effective agglomeration position versus proportion of quarterwave, and compares them to the these modelled results. The error bars show only the measurement error in the height. The results show good agreement with the modelling. Repeating the experiment, it was found that beads in the same lateral location return consistently to the same height.

In order to test mode-switching in a flow-through device, a second chamber was tested and this is shown diagrammatically in Figure 9. This chamber is also constructed from Macor and incorporates a glass slide as the reflector layer. Parameters of this device are shown in Table 1 in the column headed "Flow-through Chamber". To estimate the agglomeration position a microscope is used to look through the glass slide above the outlet, and is focussed on a plane approximately half way down the outlet channel. Since laminar flow preserves the flow structure, the position of the beads in the outlet will reflect the height they were moved to by the ultrasonic wave.

Figure 10 shows the beads being moved to a range of different effective agglomeration positions in the flow through device. The beads are a mixture of 10 and $20 \mu \mathrm{m}$ diameter of the type described above. Using the different bead sizes was intended to verify that the final position of the beads is independent of the bead size, however it is found that the sedimentation that occurs between manipulation and viewing the beads in the outlet channel causes them to be found at different positions (see below).

In Figure 10a, excitation is almost entirely via the half-wavelength mode and particles are collected at the centre of the outlet, showing they were agglomerated in the centre of the channel. As the percentage of quarter-wavelength excitation increases, particles move towards the right of the outlet until in Figure 10d the beads have been 
moved to the right hand side (see below for the effect of gravity) of the outlet, which corresponds to the beads having been near the glass slide side of the fluid channel as they flowed over the transducer. It can be seen that as the proportion of quarter-wave mode is increased that the agglomeration position shifts towards this height in the channel. It should be noted that particles sediment a little under the action of gravity in the region of the channel between the end of the transducer and the outlet, so the position of the beads in the outlet does not correspond exactly to the position of the beads in the channel; nevertheless it provides a good demonstration of the consistent control over particle position provided by mode-switching. The amount of sedimentation that occurs is inversely proportional to the flow-rate: an increased flow rate will allow less time for sedimentation. In this experiment the flow rate was $1.8 \mathrm{~mm} . \mathrm{s}^{-1}$ in the chamber, which means that a $20 \mu \mathrm{m}$ bead (density $1055 \mathrm{~kg} \cdot \mathrm{m}^{-3}$ ) can be expected, from equation (5), to sediment a distance of approximately $14 \mu \mathrm{m}$ in the $2 \mathrm{~mm}$ between the edge of the transducer and the outlet, though uncertainties over the strength of the acoustic field near the edge of the transducer limit the accuracy of this calculation.

\section{Conclusions}

It has been shown that it is possible to control the agglomeration position in a multimode chamber by switching in a controlled manner between those modes. Mode-switching offers a predictable and effective means of positioning particles at any arbitrary position between the half and quarter-wave node positions in an ultrasonic particle manipulator. The approach has been demonstrated in two chambers both with and without flow.

Agglomeration position is sensitive to variations in the relative strengths of the two modes and some lateral variations are observed. However, this approach allows a "fine tuning” of ultrasonic manipulation while maintaining resonant (and hence efficient) operation. Potential advantages offered by such control include the ability to compensate for design tolerances in resonant chambers and the potential to maintain precise positioning of particles despite changes in temperature and/or suspending fluid characteristics.

\section{References}

[1] M. Gröschl, "Ultrasonic separation of suspended particles - Part I: Fundamentals," Acustica, vol. 84, pp. 432-447, 1998.

[2] N. R. Harris, M. Hill, S. P. Beeby, Y. Shen, N. M. White, J. J. Hawkes, and W. T. Coakley, "A Silicon Microfluidic Ultrasonic Separator," Sensors and Actuators B, vol. 95, pp. 425-34, 2003.

[3] J. J. Hawkes, R. W. Barber, D. R. Emerson, and W. T. Coakley, "Continuous cell washing and mixing driven by an ultrasound standing wave within a microfluidic channel," Lab On A Chip, vol. 4, pp. 446-452, 2004.

[4] T. Masudo and T. Okada, "Particle separation with ultrasound radiation force," Current Analytical Chemistry, vol. 2, pp. 213-227, 2006.

[5] F. Petersson, L. Åberg, A.-M. Swärd-Nilsson, and T. Laurell, "Free Flow Acoustophoresis: Microfluidic-Based Mode of Particle and Cell Separation," Anal. Chem., vol. 79, pp. 5117-23, 2007.

[6] D. Bazou, L. A. Kuznetsova, and W. T. Coakley, "Physical environment of 2-D animal cell aggregates formed in a short pathlength ultrasound standing wave trap," Ultrasound In Medicine And Biology, vol. 31, pp. 423-430, 2005. 
[7] L. A. Kuznetsova and W. T. Coakley, "Applications of ultrasound streaming and radiation force in biosensors," Biosensors \& Bioelectronics, vol. 22, pp. 1567-1577 2007.

[8] S. P. Martin, R. J. Townsend, L. A. Kuznetsova, K. A. J. Borthwick, M. Hill, M. B. McDonnell, and W. T. Coakley, "Spore and micro-particle capture on an immunosensor surface in an ultrasound standing wave system," Biosensors \& Bioelectronics, vol. 21, pp. 758-767, 2005.

[9] T. J. Mason, "Developments in ultrasound-Non-medical " Progress in Biophysics \& Molecular Biology, vol. 93, pp. 166-175 2007.

[10] H. Bohm, P. Anthony, M. R. Davey, L. G. Briarty, J. B. Power, K. C. Lowe, E. Benes, and M. Groschl, "Viability of plant cell suspensions exposed to homogeneous ultrasonic fields of different energy density and wave type," Ultrasonics, vol. 38, pp. 629-632, 2000.

[11] Z. W. Wang, P. Grabenstetter, D. L. Feke, and J. M. Belovich, "Retention and viability characteristics of mammalian cells in an acoustically driven polymer mesh," Biotechnology Progress, vol. 20, pp. 384-387, 2004.

[12] J. Hultström, O. Manneberg, K. Dopf, H. M. Hertz, H. Brismar, and M. Wiklund, "Proliferation and viability of adherent cells manipulated by standing-wave ultrasound in a microfluidic chip," Ultrasound Med. Biol.. vol. 33, pp. 175-81, 2006.

[13] M. Wiklund, J. Toivonen, M. Tirri, P. Hanninen, and H. M. Hertz, "Ultrasonic enrichment of microspheres for ultrasensitive biomedical analysis in confocal laserscanning fluorescence detection," Journal Of Applied Physics, vol. 96, pp. 12421248, 2004.

[14] T. Lilliehorn, U. Simu, M. Nilsson, M. Almqvist, T. Stepinski, T. Laurell, J. Nilsson, and S. Johansson, "Trapping of microparticles in the near field of an ultrasonic transducer," Ultrasonics, vol. 43, pp. 293-303, 2005.

[15] Y. Abe, M. Kawaji, and T. Watanabe, "Study on the bubble motion control by ultrasonic wave," Experimental Thermal and Fluid Science, vol. 26, pp. 817-826, 2002.

[16] A. Neild, S. Oberti, F. Beyeler, J. Dual, and B. J. Nelson, "A micro-particle positioning technique combining an ultrasonic manipulator and a microgripper," Journal of Micromechanics And Microengineering, vol. 16, pp. 1562-1570, 2006.

[17] J. J. Hawkes and W. T. Coakley, "Force field particle filter, combining ultrasound standing waves and laminar flow," Sensors and Actuators B-Chemical, vol. 75, pp. 213-222, 2001.

[18] M. Hill, Y. Shen, and J. J. Hawkes, "Modelling of layered resonators for ultrasonic separation.," Ultrasonics, vol. 40, pp. 385-92, 2002.

[19] H. Bohm, L. G. Briarty, K. C. Lowe, J. B. Power, E. Benes, and M. R. Davey, "Quantification of a novel h-shaped ultrasonic resonator for separation of biomaterials under terrestrial gravity and microgravity conditions," Biotechnology And Bioengineering, vol. 82, pp. 74-85, 2003.

[20] F. Petersson, A. Nilsson, C. Holm, H. Jonsson, and T. Laurell, "Continuous separation of lipid particles from erythrocytes by means of laminar flow and acoustic standing wave forces," Lab On A Chip, vol. 5, pp. 20-22, 2005.

[21] H. Nowotny, E. Benes, and M. Schmid, "Layered Piezoelectric Resonators with an Arbitrary Number of Electrodes (General One-Dimensional Treatment)," Journal of the Acoustical Society of America, vol. 90, pp. 1238-1245, 1991. 
[22] M. Hill, "The selection of layer thicknesses to control acoustic radiation force profiles in layered resonators," Journal of the Acoustical Society of America, vol. 114, pp. 2654-2661, 2003.

[23] N. R. Harris, M. Hill, Y. Shen, R. J. Townsend, S. P. Beeby, and N. M. White, "A dual frequency, ultrasonic, microengineered particle manipulator," Ultrasonics, vol. 42, pp. 139-44, 2004.

[24] M. Hill, R. J. Townsend, and N. R. Harris, "Modelling for the robust design of layered resonators for ultrasonic particle manipulation," Submitted to Ultrasonics, 2008.

[25] F. Petersson, "On acoustic particle and cell manipulation in microfluidic systems," in Department of Electrical Measurements. vol. PhD: Lund University, 2007.

[26] O. Manneberg, B. Vanherberghen, B. Onfelt, and M. Wiklund, "Flow-free transport of cells in microchannels by frequency-modulated ultrasound," Lab on a Chip, vol. 9, pp. 833-837, 2009.

[27] R. J. Townsend, M. Hill, N. R. Harris, and N. M. White, "Investigation of twodimensional acoustic resonant modes in a particle separator," Ultrasonics, vol. 44, pp. e467-e471, 2006.

[28] S. M. Hagsater, T. G. Jensen, H. Bruus, and J. P. Kutter, "Acoustic resonances in microfluidic chips: full-image micro-PIV experiments and numerical simulations," Lab on a Chip, vol. 7, pp. 1336-1344, 2007.

[29] O. Manneberg, J. Svennebring, H. M. Hertz, and M. Wiklund, "Wedge transducer design for two-dimensional ultrasonic manipulation in a microfluidic chip," Journal of Micromechanics and Microengineering, vol. 18, pp. -, 2008. 


\section{Figure captions}

Figure 1: A one-dimensional, multi-layer structure

Figure 2: Force profiles in the fluid layer for idealised quarter and half-wave modes. Circles show equilibrium positions of a particle in the absence of other forces.

Figure 3: Simulated time-averaged force profiles $\left(F_{Q H}\right)$ with differing fractions of quarter wave mode. Circles show the effective equilibrium position of a particle in the acoustic field in the absence of other forces.

Figure 4: Particle agglomeration positions for varying proportions of quarter wave. In each case the quarter wave mode has force amplitude $1 \mathrm{pN}$. The half-wave mode has amplitudes $1 \mathrm{pN}, 2 \mathrm{pN}, 8 \mathrm{pN}$, and $2 \mathrm{pN}$ for (a),(b),(c),(d) respectively. The effect of a $0.1 \mathrm{pN}$ gravitational force is included for (d)

Figure 5: The circular microfluidic chamber

Figure 6: Modelled acoustic pressure amplitude through the device for (a) half-wave and (b) quarter-wave modes. Left hand side corresponds to the transducer/carrier layer boundary, and right-hand side to the reflector air boundary.

Figure 7: Modelled force profiles in the device

Figure 8: Bead height (agglomeration position) vs $\alpha$. Experimental results compared to modelled predictions (solid line).

Figure 9: Flow-through chamber

Figure 10: Mode-switching in the flow-through chamber (annotations show percentage of quarter-wave mode).

\section{Table Caption}

Table 1: Key device parameters (mm) 
Figure 1: A one-dimensional, multi-layer structure

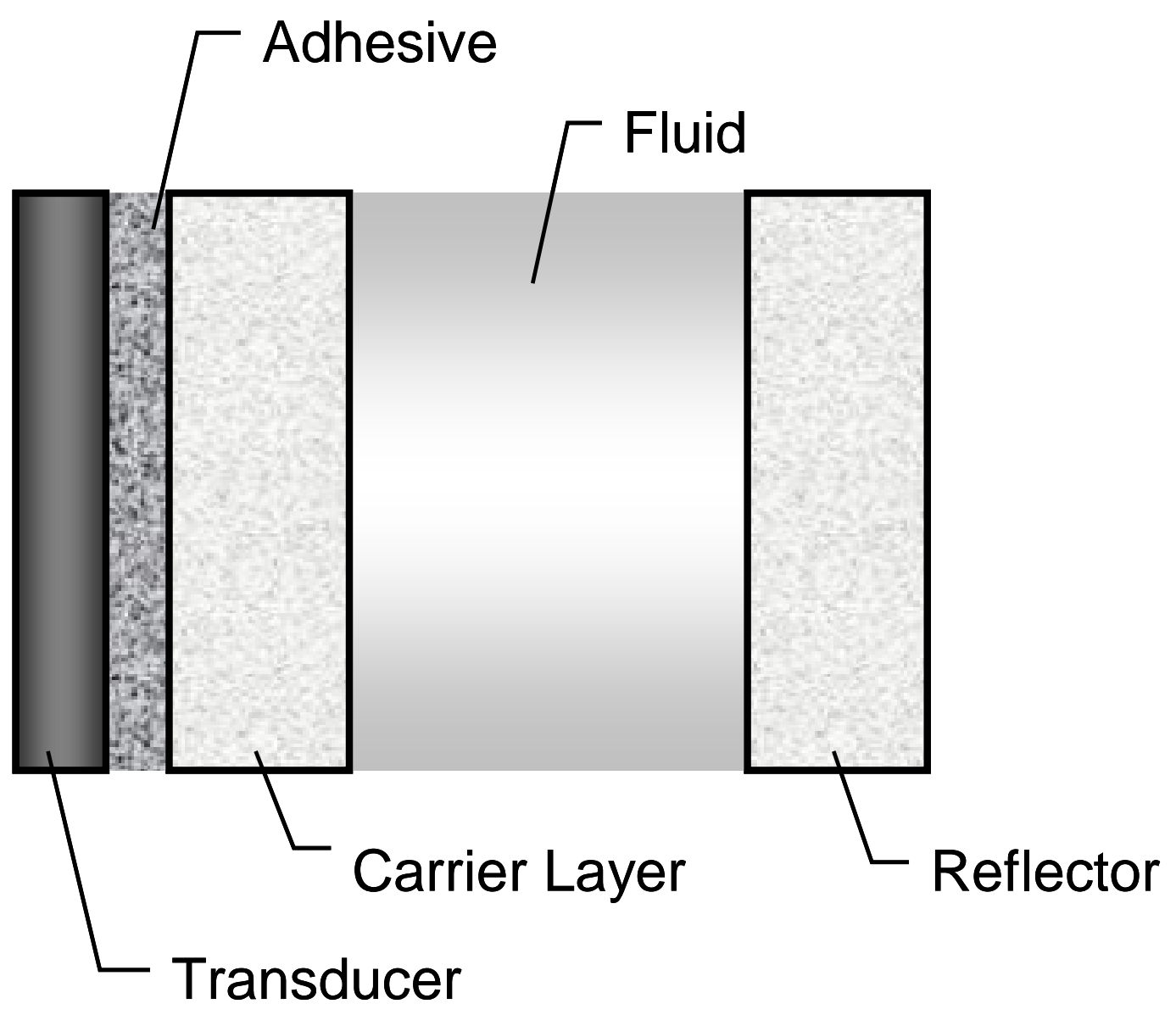


Figure 2: Force profiles in the fluid layer for idealised quarter and half-wave modes. Circles show equilibrium positions of a particle in the absence of other forces.
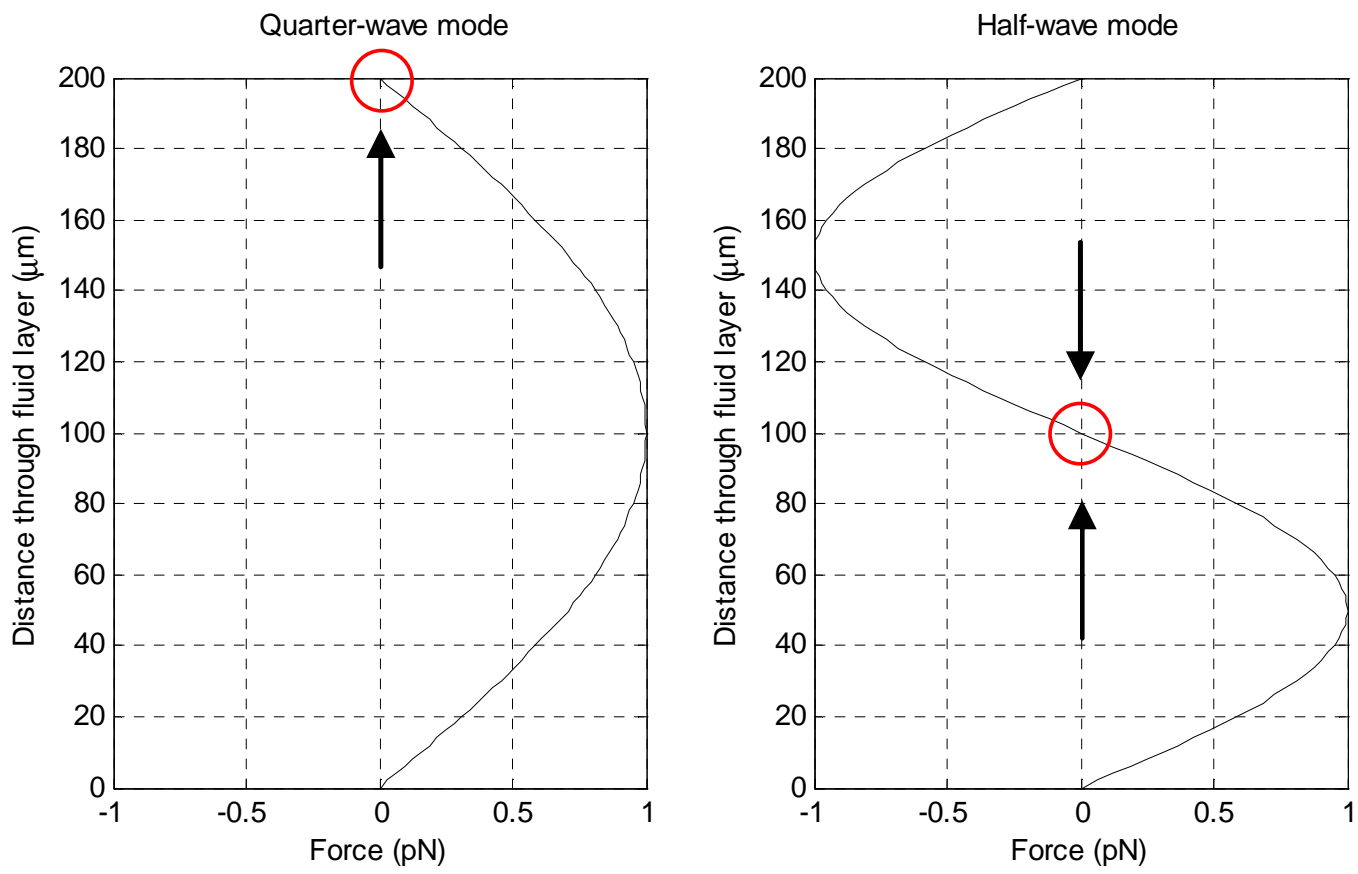
Figure 3: Simulated time-averaged force profiles $\left(F_{Q H}\right)$ with differing fractions of quarter wave mode. Circles show the effective equilibrium position of a particle in the acoustic field in the absence of other forces.
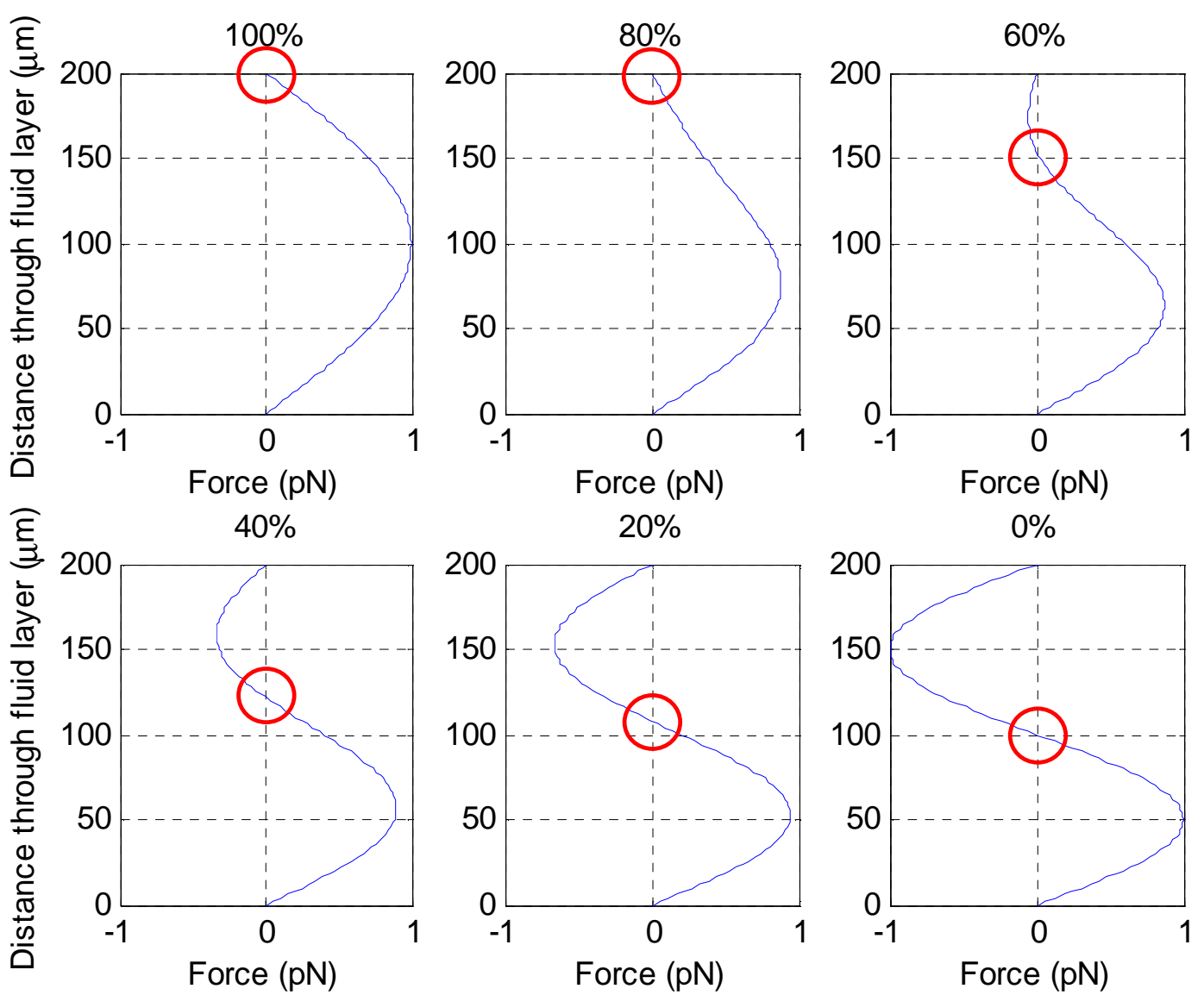
Figure 4: Particle agglomeration positions for varying proportions of quarter wave. In each case the quarter wave mode has force amplitude $1 \mathrm{pN}$. The half-wave mode has amplitudes $1 \mathrm{pN}, 2 \mathrm{pN}, 8 \mathrm{pN}$, and $2 \mathrm{pN}$ for (a),(b),(c),(d) respectively. The effect of a $0.1 \mathrm{pN}$ gravitational force is included for $(\mathrm{d})$

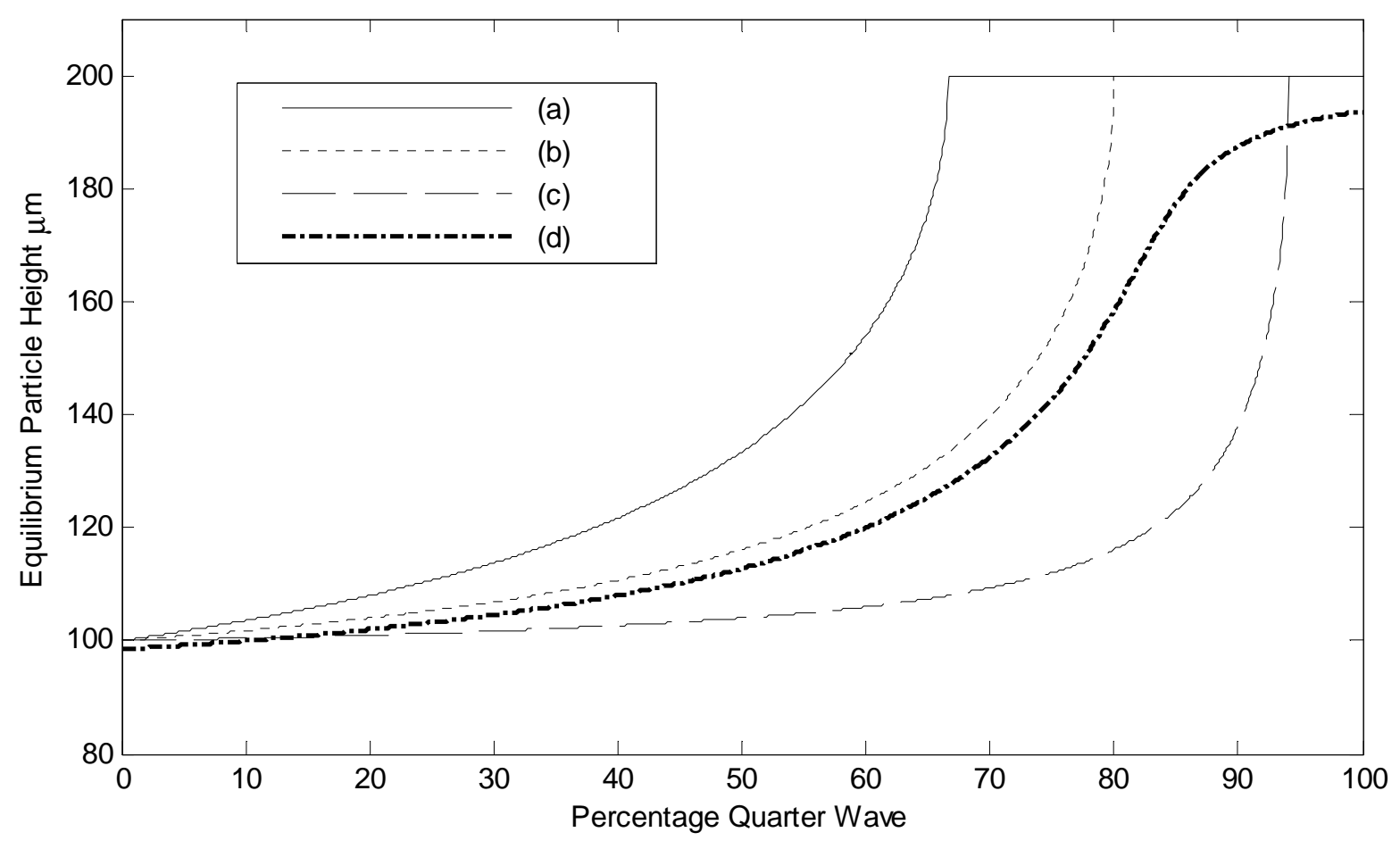


Figure 5: The microfluidic chamber
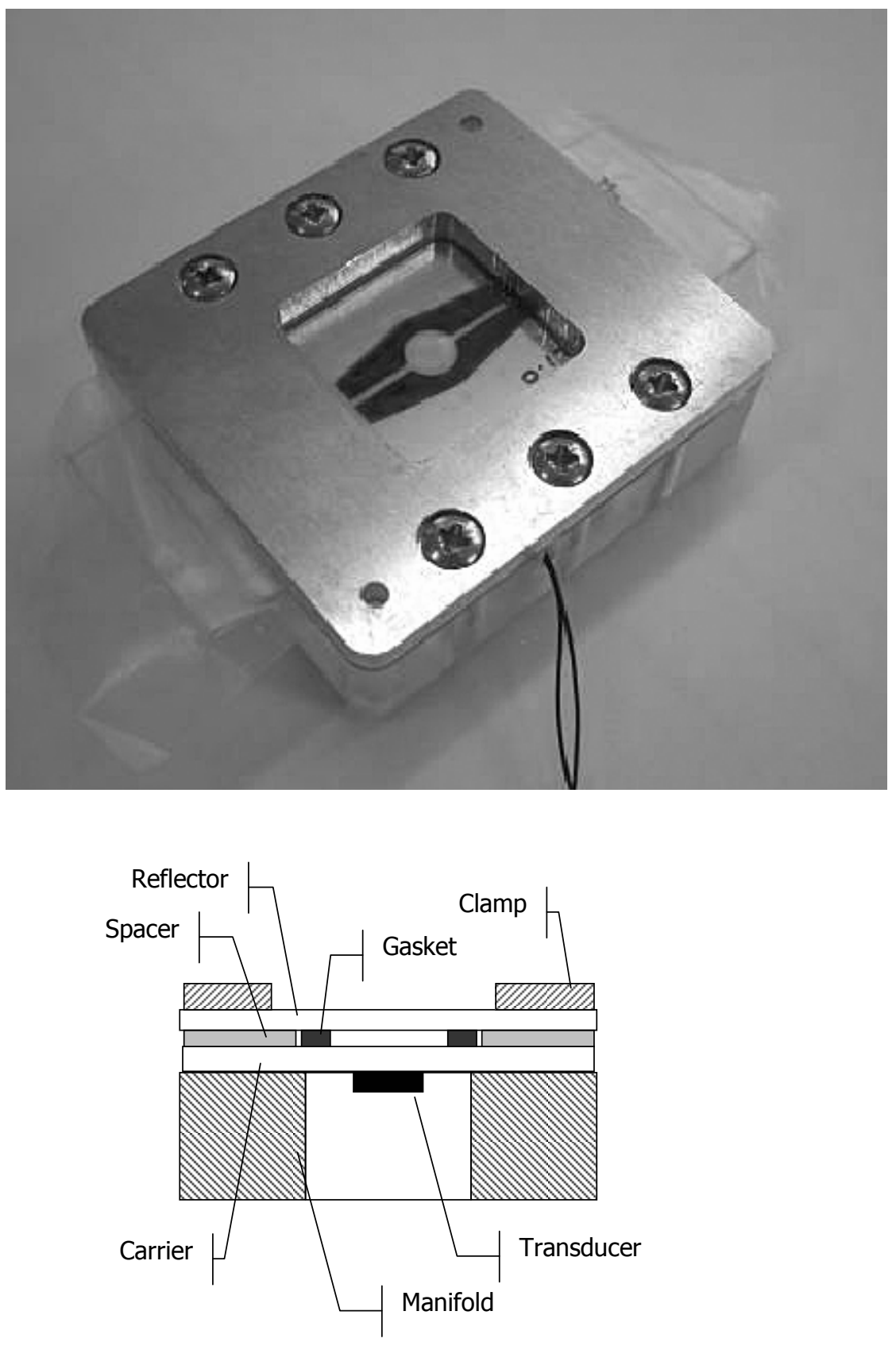
Figure 6: Modelled acoustic pressure amplitude through the device for (a) half-wave and (b) quarter-wave modes. Left hand side corresponds to the transducer/carrier layer boundary, and right-hand side to the reflector air boundary.

(a)

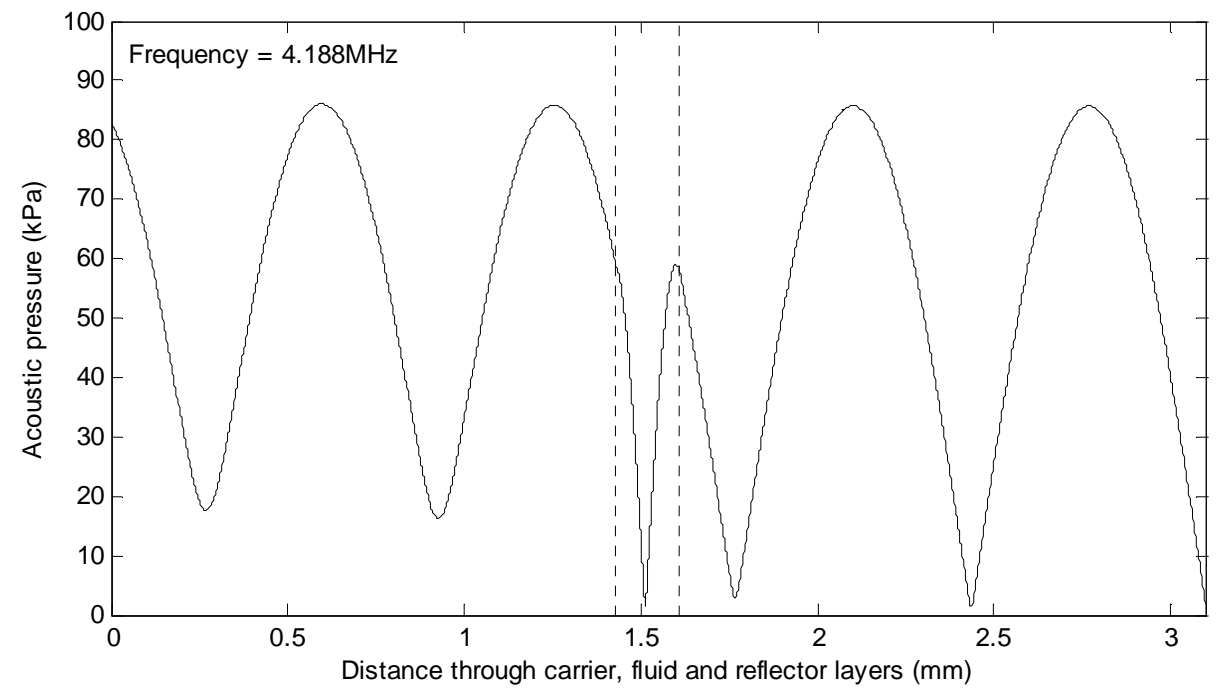

(b)

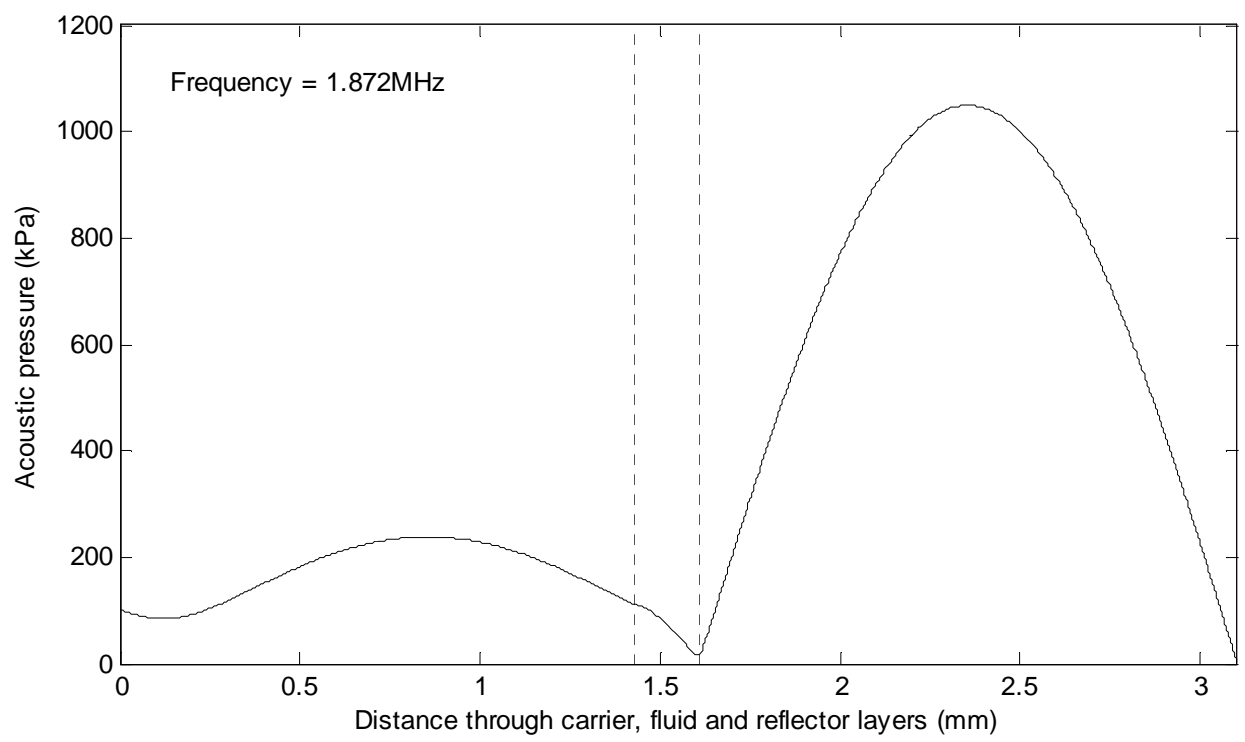


Figure 7: Modelled force profiles in the device
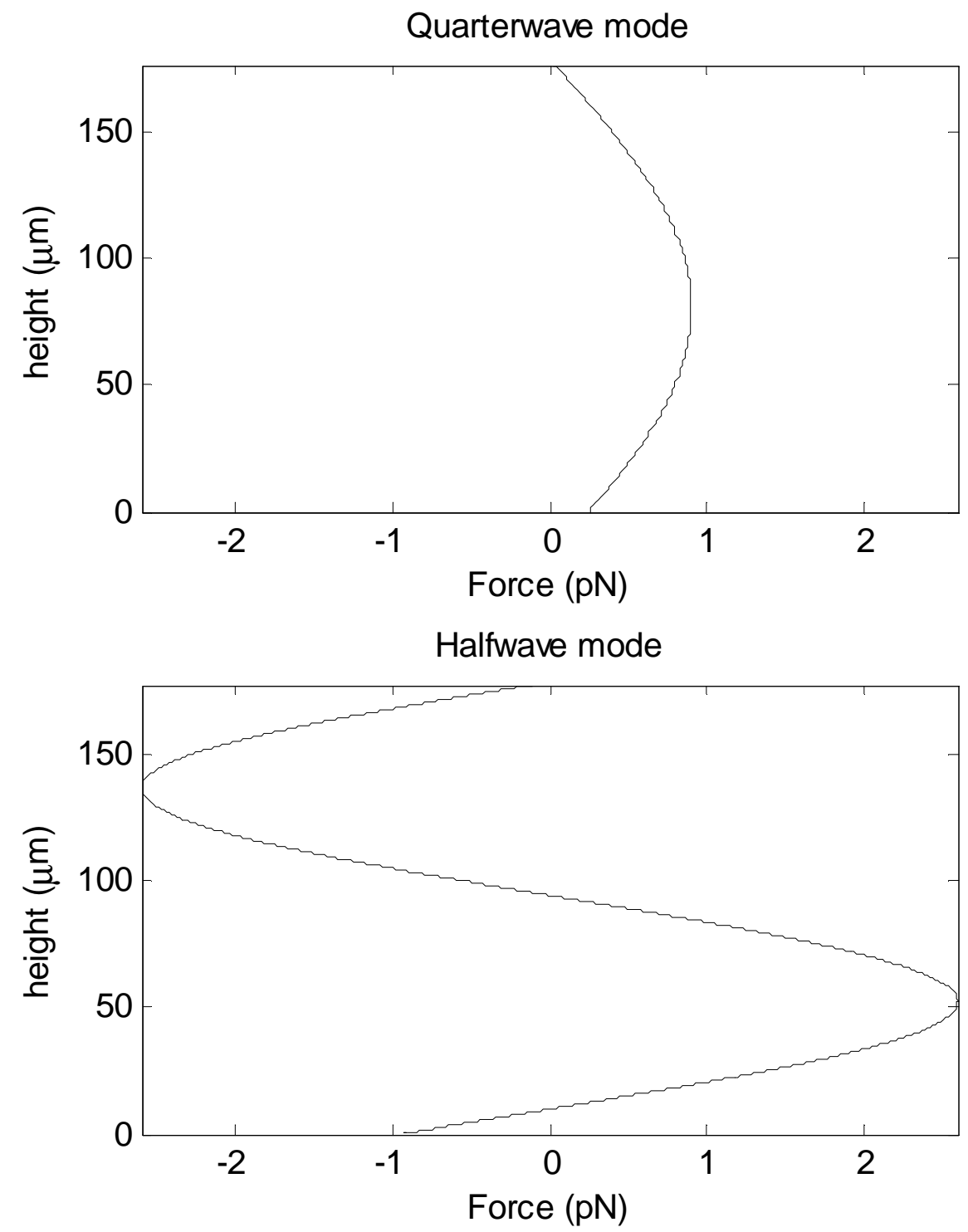
Figure 8: Bead height (agglomeration position) vs $\alpha$. Experimental results compared to modelled predictions (solid line).

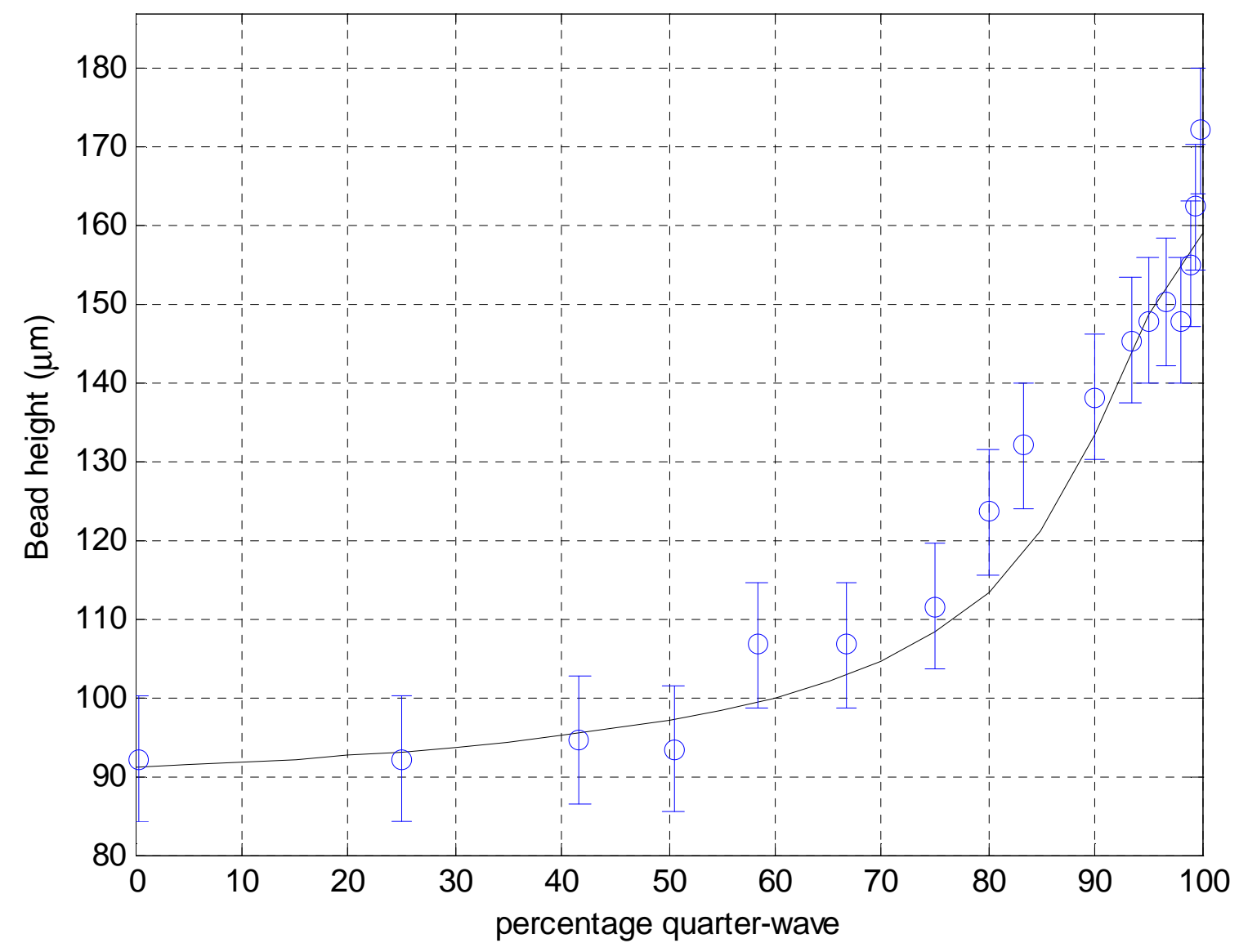


Figure 9: Flow-through chamber

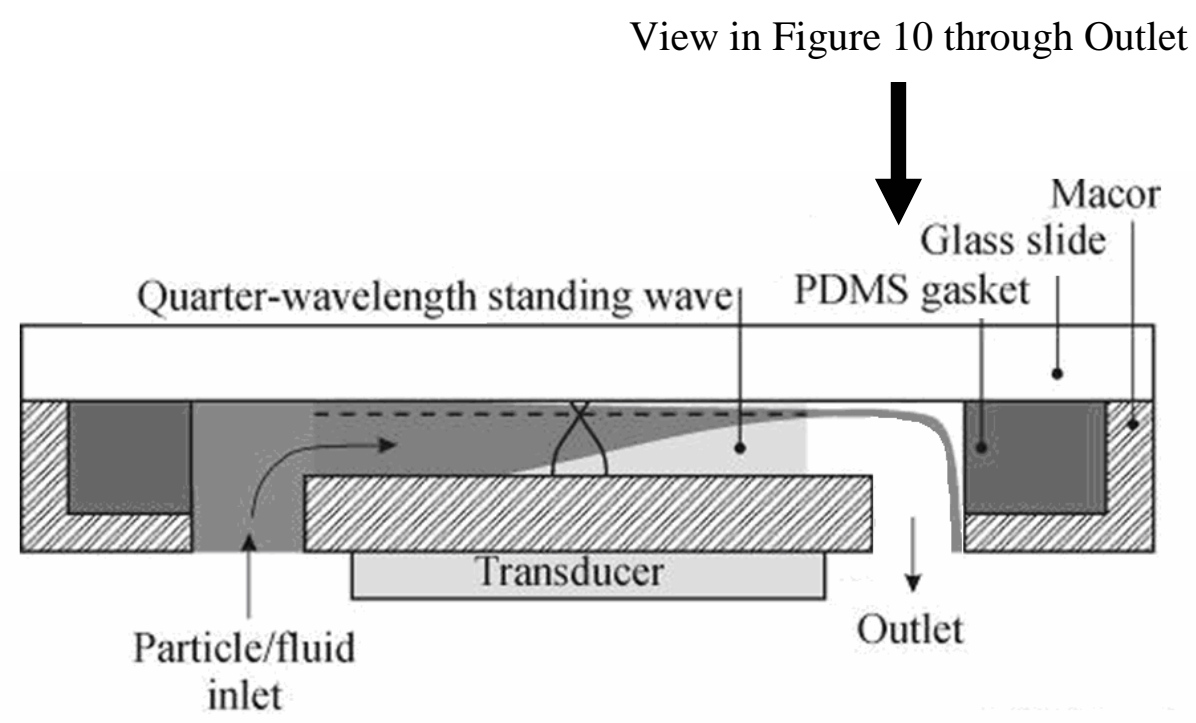


Figure 10: Mode-switching in the flow-through chamber (annotations show percentage of quarter-wave mode).

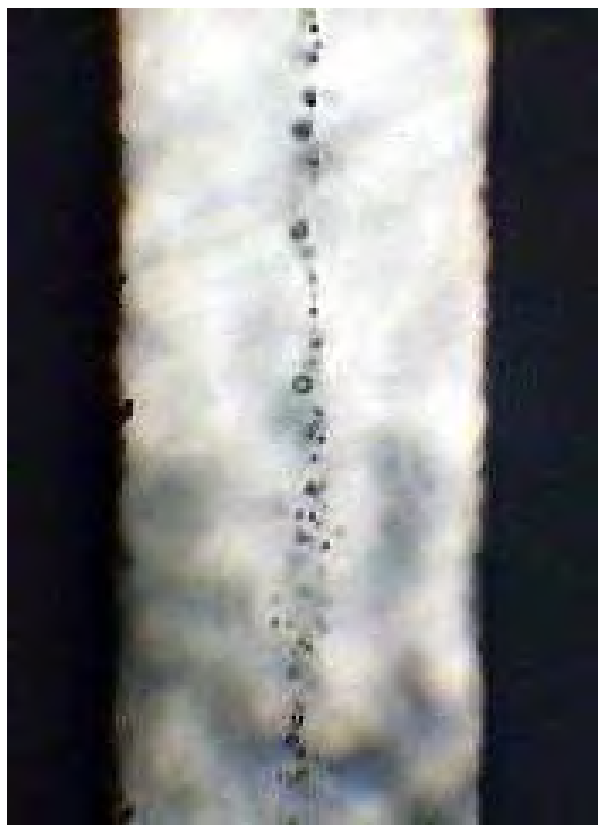

(a) $1 \%$

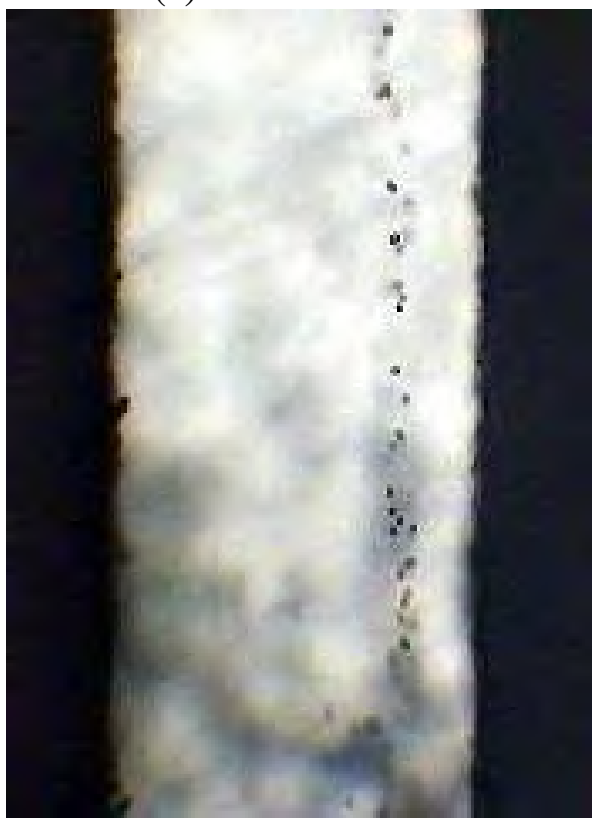

(c) $50 \%$

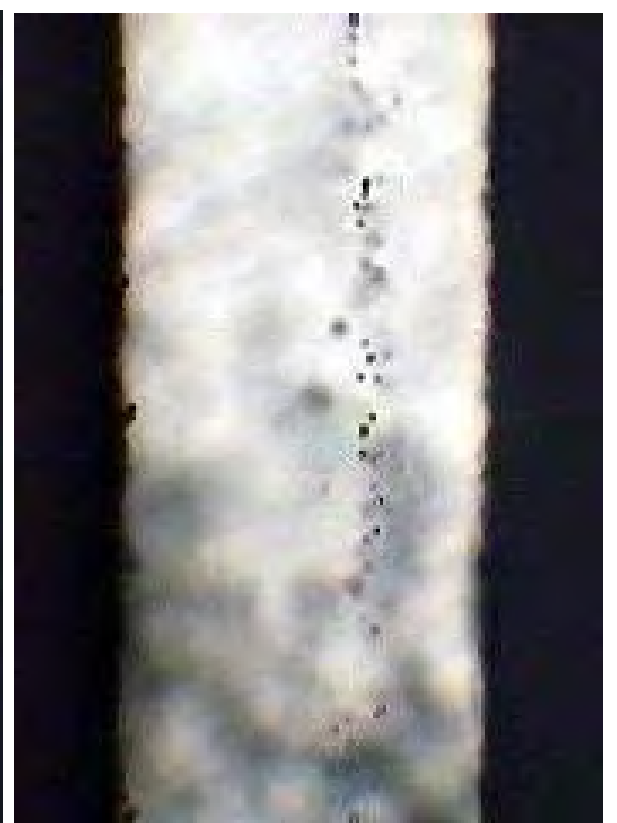

(b) $25 \%$

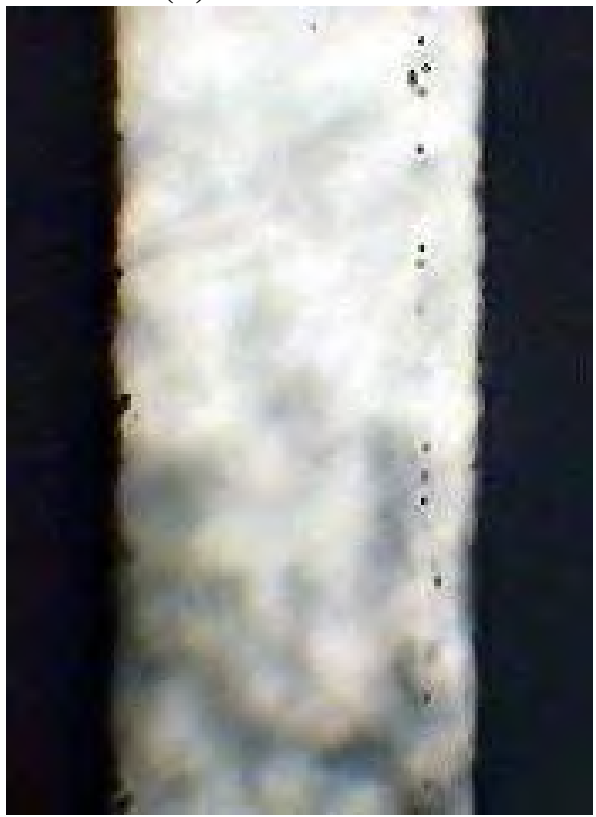

(d) $99 \%$ 